" primer le droit de propriété privée, mais il peut en "régler et en restreindre l'exercice en vue d'un intérêt " général. "

Le tribunal des conflits a suivi, dans sa décision, la thèse du commissaire du gouvernement, et il a déclaré que si bien la Cour d'appel avait eu raison de se prononcer sur le caractère privé de la propriété revendiquée par Décamps, elle n'avait pas eu moins raison de déclarer qu'elle ne pouvait, par voie de conséquence, interdire au préfet d'exercer sur cette propriété, c'est-à-dire sur l'étang, un droit de police (I).

On pourrait citer d'autres arrêts dans le même sens, mais ce serait inutile. Il est évident par exemple que les pouvoirs du préfet, si une usine est située sur un cours d'eau rattaché à un étang, iront jusqu'à en réglementer la hauteur de la retenue pour fixer le niveau conformément aux intérêts de tous. Et c'est peut-être le côté le plus intéressant de la question.

Paul Bougault, A vocat à la Cour dappel de Lyon.

\section{Nouveau mode d'application \\ du tube de Pitot-Darcy à la mesure de la vitesse des conduites d'eau sous pression}

Note de M. H. Bellet, à l'Académie des Sciences (2) séance du 5 Juin rgo5.

Pour pouvoir déterminer le rendement d'un moteur hydraulique alimenté par une conduite forcée, il est nécessaire de pouvoir mesurer aussi exactement que possible le débit de cette conduite.

Voici un nouveau dispositif qui, adapté au tube de PitotDarcy, permet de mesurer la vitesse avec une très grande approximation. Il est basé sur le même principe que l'appareil enrégistreur dont M. Mesnager a entretenu l'Académie dans sa Note du I I janvier 1904 (3); mais il est plus sensible, car il peut déceler une différence de pression pouvant être inférieure à I/Io de millimètre d'eau. Il consiste à relier l'un des ajutages du PitotDarcy à l'une des branches d'un tube en $U$, non capillaire, dans lequel on a préalablement versé un liquide $\mathrm{A}$, non miscible à l'eau et un peu plus dense que celle-ci, et à relier l'autre ajutage à l'autre branche de l' $U$, de manière à constituer un circuit complètement fermé.

Lorsque l'eau de la conduite est animée d'une certaine vitesse, la pression n’étant pas la même dans les deux ajutages, il se produit, dans les deux branches du tube en $U$, une dénivellation des surfaces de contact de l'eau et du liquide A. Si l'on désigne par $d$ la densité par rapport à l'eau du liquide A, et par $h$ la hauteur de la dénivellation produite, la relation qui donne la vitesse $V$ en fonction de $h$ est :

$$
k \frac{V^{2}}{2 g}=h(d-\mathrm{I})
$$

$k$ étant le coefficient pratique du Pitot-Darcy. Ce coefficient est d'ailleurs très voisin de l'unité.

On voit immédiatement que la sensibilité du manomètre

(I) Consulter aussi l'avis du Conseil d'Etaten date du 3 mars 1858 , intitulé : "Avis du Conseil d'Etat sur la question de savolr jusqu'où " s'étend la compétence administrative en matière de règlement "d'étangs ". (Dalloz, Code des lois alministratives, no 4828 , en note, et Dalloz Périodique, I892, 3, 59, en note, notes I et II).

(2) Présentée par M. Boussinesq.

(3) Voir La Hounlle Blanche, février 1904. pourra être très grande, et qu'elle le sera d'autant plus que la densité du liquide A sera elle-même plus voisine de l'unité.

Au lieu d'employer un liquide plus dense que l'eau, on peut également employer un liquide moins dense. Il suffit simple. ment de renverser le tube en $U$.

Le liquide employé A doit être non miscible à l'eau et inso. luble dans celle ci. Il ne doit pas mouiller le verre et, pour faciliter les lectures, il doit être coloré, ou bien il doit dissoudre une matière colorante insoluble dans l'eau. Tels sont, par exemple, le sulfure de carbone $(d=1,27$ environ), le tétra. chlorure de carbone $(d=1,64$ environ), la benzine $(d=0,85$ environ), les huiles de pétrole, etc.

Le sulfure de carbone, notamment, donne de bons résultats, Sa densité est voisine de 1,27 et l'on peut facilement le colorer avec l'iode, qui est insoluble dans l'eau en présence du sulfure. La séparation du sulfure d'avec l'eau se fait suivant un ménisque très marqué et elle est très nette.

Les vitesses employées dans les conduites d'eau sont généra. lement comprises entre I m. et $3 \mathrm{~ms}$ par seconde. Or la mesure de la dénivellation peut se faire à $1 \mathrm{~mm}$. près; ce qui, pour une vitesse de $\mathrm{I} \mathrm{m}$. correspond à une erreur sur la vitesse de $3 \mathrm{mms}$ seu. lement, de sorte que cette vitesse peut être estimée, abstraction faite du coefficient $k$ du Pitot-Darcy, avec une approximation atteignant 0,3 pour Ioo.

Lorsqu'on soumettra l'appareil à des pressions différentes, la densité du liquide A pourra varier légèrement; mais, sans déterminer exactement la valeur de cette densité, non plus que celle du coefficient $k$, il suffira de tarer préalablement l'appareil en laboratoire, pour un liquide donné, et de dresser une Table correspondante, pour avoir tous les éléments nécessaires à une mesure.

\section{Démarreur automatique pour moteur à courant triphasé}

Un problème intéressant, et vraiment pratique, c'est celui qui consiste à remplir un réservoir d'eau automatiquement, au moyen d'une pompe devant se mettre en marche d'elle-même lorsque le réservoir est vide et s'arrêter lorsqu'il est plein. Ces opérations doivent pouvoir s'effectuer sans aucune surveillance et l'installation ne doit nécessiter tout au plus qu'une visite tous les deux ou trois jours ou même seulement une fois par semaine pour s'assurer que tout fonctionne normalement.

Seule, ou à peu près, l'électricité peut doñner une solution heureuse à ce problème, et avec le nombre toujours croissant des distributions d'énergies électriques, surtout par courant triphasé, on peut espérer en voir bientôt de nombreuses applications. Nous croyons donc être utile à nos lecteurs en leur décrivant un démarreur automatique robuste et ayant reçu depuis quelques années déjà la sanction de la pratique.

Ce démarreur, construit par la maison Voigt et Haeffner que représentent en France MM. Richard, Ch. Heller et Cie, peut être combiné, soit pour les moteurs à courant continu, soit pour les moteurs à courant triphasé. Nous décrirons seulement en détail le dispositif employé dans ce dernier cas comme étant le plus intéressant et présentant le plus d'applications possibles; nous nous bornerons ensuite d indiquer le principe de l'appareil employé avec le courant continu. Nous supposerons, d'autre part, que le motellr triphasé a son rotor bobiné et possède des bagues pour l'insertion des résistances de démarrage. On verra ensuité quelle simplicité se réduit l'appareil lorsqu'il est possible d'utiliser un moteur avec induit en cage d'écureuil.

A la mise en marche, le démarreur automatique a uin 
double rôle à remplir, d'abord fermer l'interrupteur pour envoyer le courant dans le stator, puis supprimer, successivement et lentement, les résistances placées en série sur le rotor jusqu'à la mise en court-circuit de ce dernier. A l'arrêt, deux opérations également : ouverture de l'interrupteur du stator et suppression du court-circuit sur le rotor. Il faut, en outre, que tout soit remis en état pour une nouvelle mise en marche.

Du réservoir lui-même, souvent situé à plusieurs centaines de mètres de la pompe, un flotteur commande la mise en marche ou l'arrêt en actionnant une poulie sur laquelle est fixée une aiguille. Cette aiguille vient buter tour à tour contre deux plots suivant que le réservoir, plein ou vide, amène le flotteur en haut ou au bas de sa course.

Lorsque le réservoir est vide, l'aiguille de l'appareil des contacts se trouvant ramenée par le flotteur sur le plot de mise en marche, les conducteurs 2 et 3 (Voir fig. I le schéma des connexions ci-joint) sont alors mis en communication et
Le courant étant dans le stator, et le rotor étant fermé sur les résistances $R_{1}, R_{2}$ et $R_{3}$ placées en série, le moteur commence à démarrer. Pendant ce temps les bornes 2' et 3 ' qui communiquent entre elles par l'intermédiaire de l'interrupteur $i$ et de l'électro-aimant $B$, font que celui-ci, alors excité, actionne un appareil représenté très schématiquement sur la figure I. Cet appareil a pour but d'envoyer le courant successivement dans les électros $A_{1}, A_{2}$ et $A_{3}$ afin de mettre en court-circuit, au fur et à mesure du démarrage, les résistances $R_{1}, R_{2}$ puis $R_{3}$. Il se compose essentiellement d'une crémaillère actionnée par l'armature de l'électro et engrenant un petit pignon solidaire d'une sorte de commutateur cylindrique $C$ chargé de brancher successivement les électros $A_{1}, A_{2}$ et $A_{3}$ entre les bornes 2' et 3'. Pour permettre au démarrage de s'effectuer durant le temps voulu, on retarde le mouvement de la crémaillère en la taisant actionner une petite palette qui tourne assez rapidement et fait office d'amortisseur à air. Lorsque l'interrupteur $I_{3}$ est

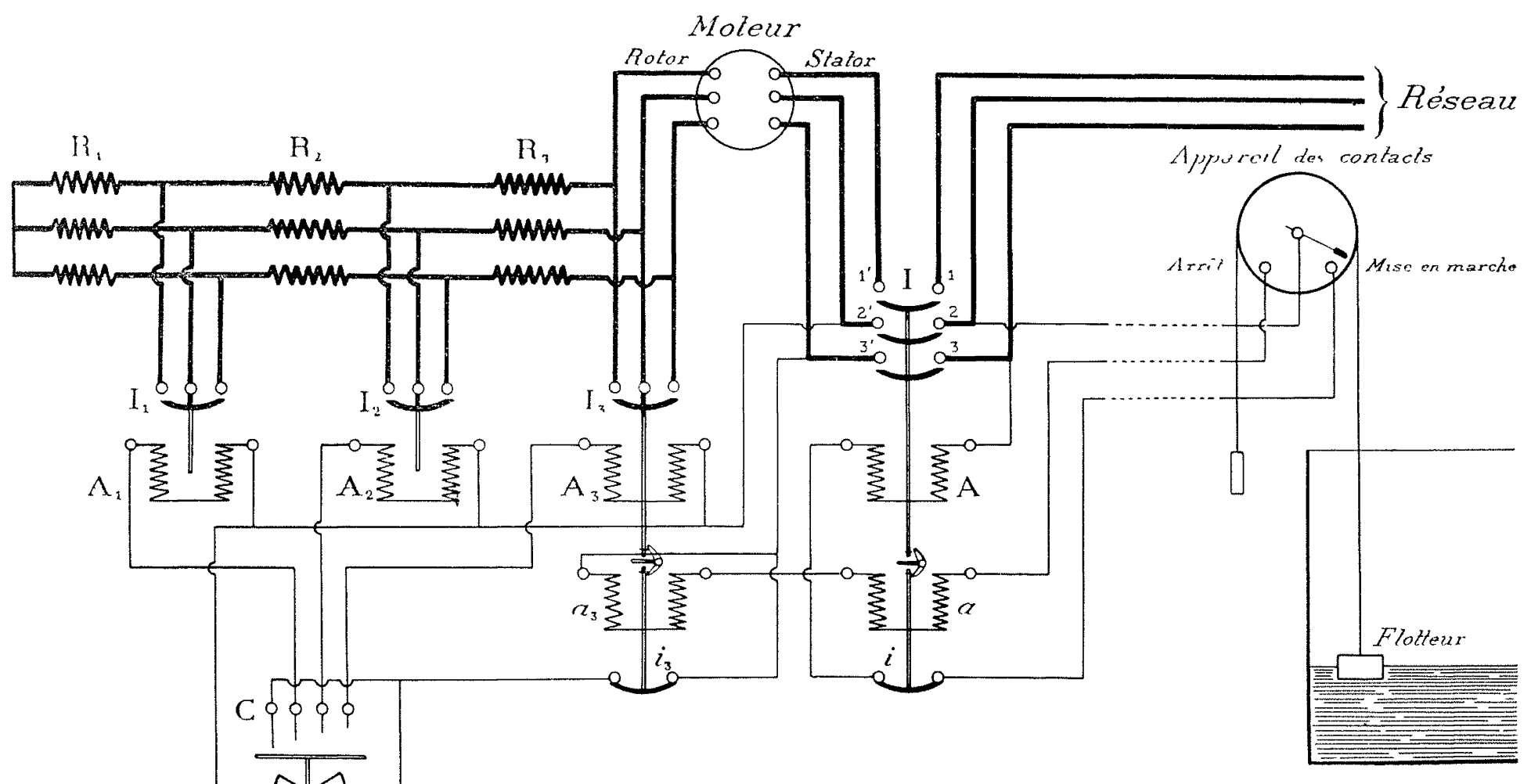

Fig. I. - Schéma général de l'installation.

forment un circuit fermé qui comprend : l'appareil des contacts, l'interrupteur $i$ enclanché à ce moment, et enfin l'électro-aimant $A$. Ce dernier, qui reçoit du courant alternatif, attire son armature et fait fermer l'interrupteur $I$ envoyant ainsi le courant triphasé dans le stator. L'inter-rupteur $I$ est alors maintenu par le double cliquet $K$ (fig 2) engagé dans l'échancrure de la tige qui supporte l'interrupteur et l'armature de l'électro $A$. Mais ce cliquet, en basculant pour venir s'engager dans l'échancrure, a dégagé la tige de l'interrupteur $i$ qui s'ouvre et interrompt le courant dans $A$. On voit, d'autre part, par les détails de la figure 2, que l'électro $a$ en attirant son armature aura pour effet d'opérer le rènversement du cliquet d'où dégagement et ouverture de l'interrupteur $I$, en même temps qu'accrochage et fermeture de l'interrupteur $i$. Ainsi ces deux interrupteurs ne peuvent rester ouverts ou fermés simultanément et l'enclanchement de l'un produit le déclanchement de l'autre ou vice versa. à la fin enclanché, le rotor étant en court-circuit, le démarrage est terminé. Comme pour les interrupteurs $I$ et $i$, une fois l'interrupteur $I_{3}$ enclanché, le petit interrupteur $i_{3}$ s'ouvre coupant le courant dans les électros $B$ et $A_{3}$. Ainsi pendant la marche du moteur, les interrupteurs $I$ et $I_{3}$ sont seuls enclanchés et aucun électro n'est sous courant.

Lorsque le réservoir est plein, l'aiguille de l'appareil des contacts venant toucher le plot de l'arrêt, les bornes 2 et 3 ' sont alors reliées ensemble par l'intermédiaire des deux électros $a$ et $a_{3}$. Ceux-ci s'excitent, font enclancher les interrupteurs $i$ et $i_{3}$ et par suite ouvrir les interrupteurs $I$ et $I_{3}$. Le moteur s'arrête donc et tout est prêt pour une nouvelle mise en marche. Remarquons que l'ouverture de l'interrupteur $I$ a pour effet de couper le courant dans les électros $a$ et $a_{3}$. Tous les électros du tableau ne sont donc sous courant que pendant le temps strictement nécessaire pour l'accomplissement de leurs fonctions.

Dans le cas d'un moteur avec induit en cage d'écu- 
reuil(1) tout le démarreur se réduit évidemment aux interrupteurs $I$ et $i$ actionnés respectivement par les électros $A$ et $a$. Le tableau devient donc très simple.

Dans le cas du courant continu le démarreur, toujours commandé par le même appareil des contacts, se compose d'un conjoncteur-disjoncteur semblable à celui que représente la figure 2 et ayant pour but d'envoyer ou de couper le courant dans le moteur. En outre une série d'électroaimants placés, par un couplage spécial, en dérivation sur l'induit du moteur, mettent en court-circuit les résistances de démarrage successivement et suivant l'accroissement de la vitesse du moteur. Pour cela, comme la tension aux
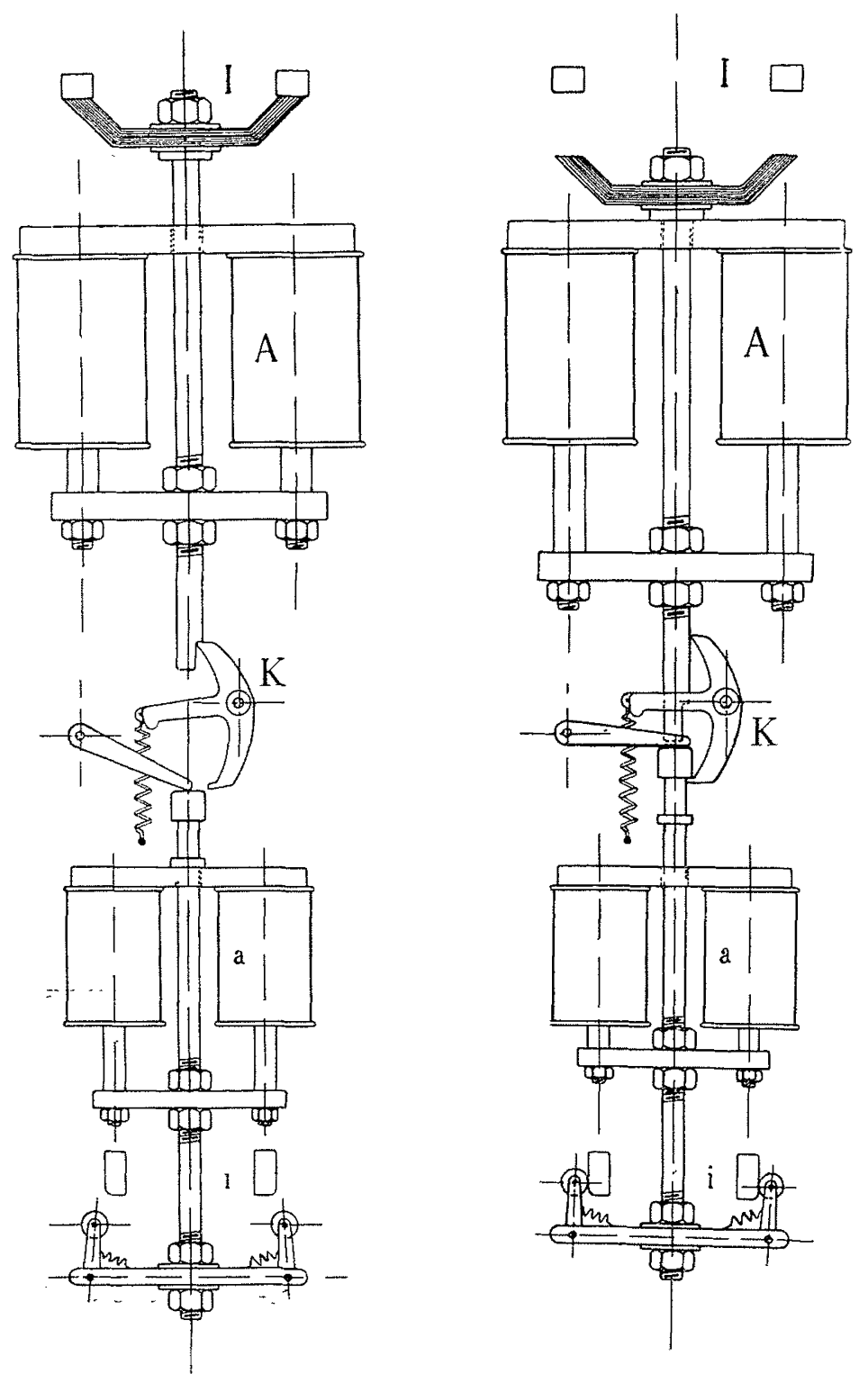

Fig. 2. - Schéma de l'appareil automatique.

bornes de l'induit croît pendant le démarrage depuis o jusqu'à la tension du réseau, les électros sont réglés pour entrer en fonction suivant différentes valeurs de la tension. $\mathrm{La}$ résistance étant judicieusement divisée il en résulte, même quand le nombre des sections est faible, un démarrage d'une régularité parfaite.

Une des applications du démarreur automatique pour courant triphasé, tel que le représente la figure (I), a été fait dernièrement à Lyon dans les sous sols du grand HôteiBellecour, par le service électrique de la Société nouvelle

(I) Ces moteurs ne sont acceptés sur la plupart des réseaux de force et d'éclairage que pour une puissance inférieure à 3 ou $4 \mathrm{chx}$. des établissements de l'Horme et de la Buire. L'installation comprend deux pompes Jandin à piston, chargées de refouler chacune $14 \mathrm{~m}^{3}$ à l'heure dans un réservoir situé à la partie supérieure de l'hôtel et destiné à fournir l'eau nécessaire au fonctionnement de l'ascenseur et des divers services de l'hôtel. La hauteur totale de refoulement, aspiration comprise, est d'environ 40 mètres. Chacune des pompes est entraînée au moyen d'une courroie par un moteur triphasé Alioth de 5 chevaux tournant à la vitesse de 960 tours par minute. Les divers appareils du démarreur automatique sont tous placés sur un tableau en marbre. Au-dessous, sur un panneau également en marbre, se trouvent une série d'interrupteurs et de commutateurs permettant de faire marcher l'une ou l'autre pompe à la main ou automatiquement. En temps ordinaire une seule pompe est en service, l'autre sert de réserve. Le démarreur actuellement fonctionne 7 à 8 fois par jour en moyenne et donne pleine satisfaction.

Cette installation, très bien aménagée, est également intéressante à un autre point de vue: Pour empêcher que le bruit des soupapes et des pistons ne se répercute dans tout l'hôtel qui, construit en maçonneries et planchers armaturés de charpentes en fer est particulièrement sonore, on a dû monter les pompes et leurs moteurs sur des bâtis en ciment armé avec des poutres de fer et reposant sur des galets en caoutchouc(I). Les tuyaux d'aspiration et de refoulement se raccordent aux pompes par des manchons également en caoutchouc. Grâce à ces précautions on est parvenu à éviter tout bruit.

Nous citerons également l'installation de pompe automa* tique que la commune de Châtillon-sur-Chalaronne, dans l'Ain, a fait effectuer il y a quelques mois. Quoique moins importante elle offre cependant beaucoup d'intérêt, car elle a pour but d'alimenter un réservoir assurant la fourniture de l'eau à cette importante commune. Là, le moteur triphasé qui sort également de l'usine Alioth, a son induit en cage d'écureuil, aussi l'installation est-elle très simple et très robuste. Etant donné le prix assez faible que coûte une semblable installation et surtout les frais d'entretien très réduits

(I) Nous croyons devoir signaler à nos lecteurs un moyen très pratique et que de nombreuses applications déjà démontrent comme étant infaillible, d'empêcher toute transmission des vibrations d'un bâti de machine au sol et aux murs des habitations. Ce moyen consiste à remplacer le mortier des massifs de fondation par de l'asphalte. On se sert pour cela de pierres convenablement appareillées et l'on maçonne comme à l'ordinaire mais en faisant les joints avec de l'asphalte préparée dans les chaudières qui servent habituellement à la confection du dallage en asphalte des trottoirs. On obtient ainsi des massifs de fondation extrêmement solides car les joints d'asphalte effectués avec les précautions voulues constituent une liaison très résistante des matériaux. Ėt, point principal, cet assemblage possède, grâce à son élasticité, un remarquable pouvoir absorbant des trépir dations. Nous en citerons comme preuve que l'on fait aujourd'hui des assises de marteaux pilons en interposant des lits d'asphalte entre les massifs de scellement des enclumes et leurs fondations en bois debout sur maçonnerie. Nous rappellerons également que c'est par l'interposition d'un lit d'asphalte entre les traverses de la voie et son assise en maçonnerie qu'à Paris, dans le prolongement jusqu'au Luxembourg de la ligne de Sceaux, l'on est parvenu à éviter radicalement la transmission à l'Observatoire des trépidations dues all passage des trains. Comme on le sait, cette vore passe en souterrain à faible distance des caves de l'Observatoire; il s'agissait dans ce cas de mettre de la manière la plus certaine cet établissement à l'abri de toute perturbation.

On peut donc éviter, ainsi très simplement, dans les locaux oú l'on veut installer des machines, ces vibrations si incommodantes et qui sont causes de tant de conflits entre cohabitants des immeubles industriels.
N. D. L. R. 
qu'elle nécessite puisque la présence d'un homme n'est plus nécessaire, on peut présumer que l'exemple donné par la commune de Chàtillon-sur-Chalaronne sera suivi par beaucoup d'autres.

Ajoutons, en terminant, que ce démarreur automatique peut trouver beaucoup d'autres utilisations en dehors de son application aux pompes et qu'il pourra rendre de très grands services chaque fois que l'on aura en vue une installation automatique quelconque dont l'arrêt ou la mise en marche devra être effectué par un simple curseur venant tour à tour en contact avec deux plots (commande automatique de la pompe d'alimentation d'une chaudière par exemple). Ce même démarreur pourra enfin être utilisé avec succès pour la mise en marche, ou l'arrêt, de moteurs placés àdıstance ou dans des endroits inaccessibles. L'appareil des contacts devient alors un simple commutateur à deux directions, l'une pour la marche, l'autre pour l'arrêt.

P. Bfrgeon, Ingénieur électricien.

\section{Remarques au sujjet de l'emploi de la méthode de M. Alliévi pour le calcul des coups de bélier}

Dans la méthode de $M$. Alliévi, si $l$ est la longueur de la conduite et $a$ la vitesse de propagation $\left(^{*}\right)$, on doit considérer des périodes successives de longueur égale à $\frac{2 l}{a}$. Si alors $Y$ et $V$ sont la pression et la vitesse dans le tuyau à l'extrémité du côté du distributeur, on a pendant la première période de longueur $\frac{2 l}{a}$ :

$$
\begin{gathered}
Y=y_{0}+F(t) \\
V=\nu_{0}-\frac{g}{a} F(t)=\nu_{0} \lambda_{1} \sqrt{\frac{\bar{Y}}{y_{0}}}
\end{gathered}
$$

où $\gamma_{0}$ et $\nu_{0}$ sont les valeurs de $Y$ et $V$ à l'état de régime, avant la mise en mouvement du distributeur, et $\lambda_{1}$ le rapport entre l'ouverture du distributeur à un instant quelconque de cette période, à son ouverture à l'état de régime lorsque la vitesse est $\nu_{0}$. Ces trois équations font connaître $Y, V$, $F(t)$ pendant la première période.

On a ensuite pour une période quelconque:

$$
\begin{gathered}
Y=y_{0}+F(t)-F\left(t-\frac{2 l}{a}\right) \\
V=\nu_{0}-\frac{g}{a}\left[F(t)+F\left(t-\frac{2 l}{a}\right)\right]=\nu_{0} \lambda_{n} \sqrt{\frac{Y}{y_{0}}}
\end{gathered}
$$

où $\lambda_{n}$ est le rapport de l'ouverture du distributeur à un instant quelconque de la $n$ e période que nous considérons, à son ouverture, à l'état de régime.

Ces trois équations, où $F\left(t-\frac{2 l}{a}\right)$ est connu au moyen

(") D'après M. Alliévi :

$$
a=\frac{9,900}{\sqrt{4^{8,3+K \frac{d}{e}}}}
$$

$d$ diamètre, e épaisseur du tuyau. $K=0,5$ pour le fer et l'acier, et
$K=1$ pour la fonte. des équations semblables relatives à la période précédente, feront connaître $Y, V$ et $F(t)$ pendant la période que nous considérons.

La méthode que nous venons de rappeler est celle due à M. Alliévi, mais elle est le plus souvent susceptible d'une très importante simplification.

Soit $\xi_{n}$ la valeur du coup de bélier pendant la $n^{\mathrm{e}}$ période, les équations précédentes s'écriront pour la première période: $\quad \xi_{1}=F(t)$

$$
V=\nu_{0}-\frac{g}{a} \xi_{1}=\nu_{0} \lambda_{1} \sqrt{\mathrm{I}+\frac{\xi_{1}}{y_{0}}}
$$

et pour l'une quelconque des périodes suivantes :

$$
\left.\begin{array}{c}
\xi_{n}=F(t)-F\left(t-\frac{2 l}{a}\right) \\
V=\nu_{0}-\frac{g}{a}\left[F(t)+F\left(t-\frac{2 l}{a}\right)\right]=\nu_{0} \lambda_{n} \sqrt{\mathrm{I}+\frac{\xi_{n}}{f_{0}}}
\end{array}\right\}
$$

Le plus souvent

$$
\frac{\xi_{n}}{y_{0}}
$$

sera inférieur à $\frac{\mathrm{l}}{2}$ et dans ces conditions on pourra prendre:

On a en effet :

$$
\sqrt{\mathrm{I}+\frac{\xi_{n}}{y_{0}}}=\mathrm{I}+\frac{\xi_{n}}{2 y_{0}}
$$

$$
\begin{aligned}
& \mathrm{I}+\frac{\mathrm{I}}{4}-\sqrt{\mathrm{I}+\frac{\mathrm{I}}{2}}=0,02525 \\
& \mathrm{I}-\frac{\mathrm{I}}{4}-\sqrt{\mathrm{I}-\frac{\mathrm{I}}{2}}=0,04289
\end{aligned}
$$

et cette différence peut être négligée, vu le degré d'approximation qu'il s'agit d'obtenir.

On peut même remarquer que ce que l'on a surtout intérêt à calculer, c'est le coup de bélier positif ; or si la formule :

$$
\sqrt{\mathrm{I}+\frac{\xi_{n}}{y_{0}}}=\mathrm{I}+\frac{\xi_{n}}{2 y_{0}}
$$

n'est évidemment pas applicable au coup de bélier négatif pour $\xi_{n}=y_{0}$, elle donnera encore une approximation, somme toute suffisante, pour le coup de bélier positif, car on a :

$$
\mathrm{I}+\frac{\mathrm{I}}{2}-\sqrt{\mathrm{I}+\mathrm{I}}=0,08579
$$

de sorte que la différence n'atteint pas $\frac{\mathrm{r}}{\mathrm{I} O}$ et que sa valeur relative est seulement de 0,06 environ. Nous prendrons donc :

$$
\sqrt{\mathrm{x}+\frac{\xi_{n}}{y_{0}}}=\mathrm{I}+\frac{\xi_{n}}{2 y_{0}}
$$

et d'après ce qui précède cette substitution sera légitime, dans le cas général, pour $\xi_{n} \leqslant \frac{y_{0}}{2}$ et dans le cas où nous nous bornons à considérer les coups de bélier positifs, pour $\xi_{n} \leqslant y_{0}$.

Nous déduirons alors des équations (2) et (3):

$$
\begin{gathered}
\xi_{n}=F(t)-F\left(t-\frac{2 l}{a}\right) \\
F(t)+F\left(t-\frac{2 l}{a}\right)=\frac{a \nu_{0}}{g}\left[\mathrm{I}-\lambda_{n}\left(\mathrm{I}+\frac{2 \gamma_{0}}{\xi_{n}}\right)\right]
\end{gathered}
$$

